\title{
Hereditary hemorrhagic telangiectasia: transient ischemic attacks
}

\author{
Dulka Manawadu MB ChB, Dilini Vethanayagam MD, S. Nizam Ahmed MD
}

See related primer by Grand'Maison, page 833, and clinical images by Nanda and Bhatt, page 838, and by Irani and Kasmani, page 839

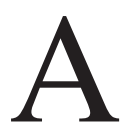
39-year-old right-handed man presented to his family physician with sudden-onset weakness in his right arm and leg that had lasted 10 minutes and resolved completely. In the year before presentation, he had an episode of weakness on the right side of his face and difficulty comprehending that had lasted for 5 minutes. The patient reported having an unexplained collapse 4 years before presentation that had not been investigated.

The patient did not smoke, and he did not use drugs or alcohol. He had no history of hypertension, diabetes, coronary artery disease or hyperlipidemia. There was no history of hemoptysis, dyspnea or fatigue. The patient reported having recurrent nosebleeds since early childhood, and his mother also had nosebleeds regularly and "low blood oxygen levels." His maternal grandmother had experienced numerous "mini-strokes."

On examination, the patient was not in distress. He had a

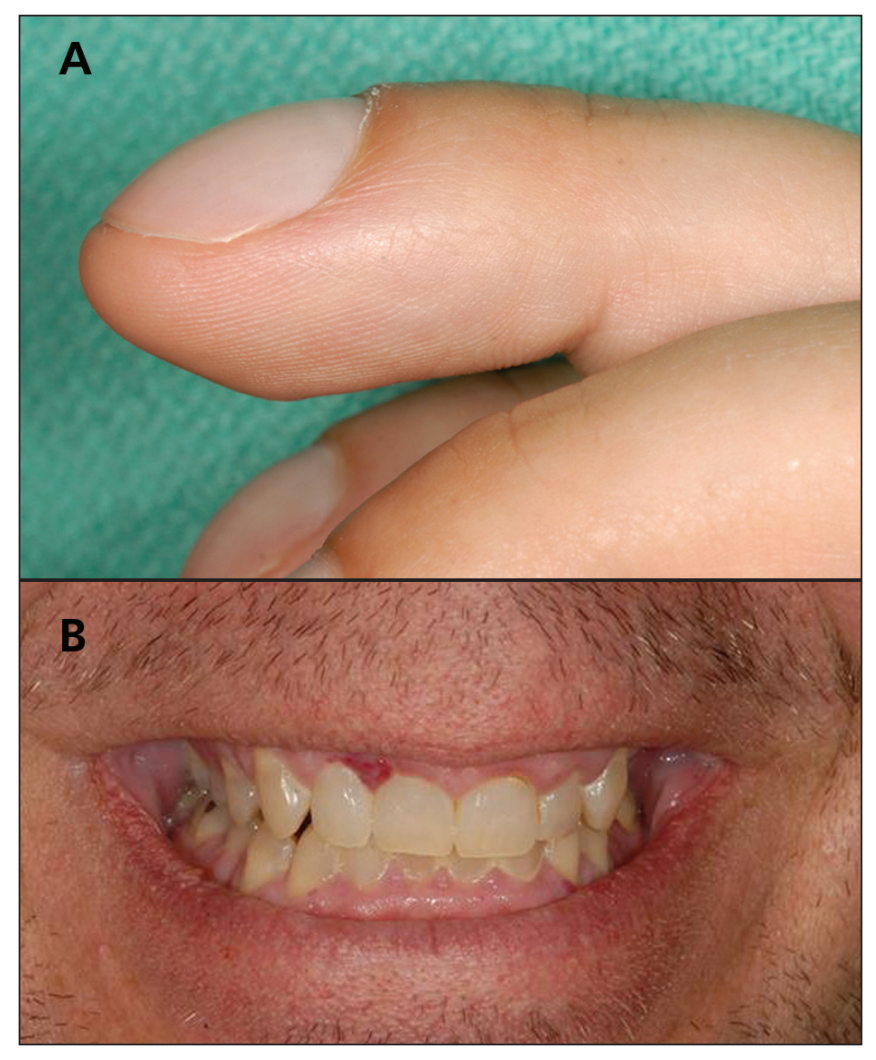

Figure 1: A 39-year-old man with clubbing of his fingers (A) and telangiectasia of the gums (B).

\section{Key points}

- Hereditary hemorrhagic telangiectasia may present with neurologic manifestations secondary to arteriovenous malformations in the lung, liver, brain or spinal cord.

- Neurologic manifestations may include headache, stroke, seizure, cerebral abscess, encephalopathy and spinal compression syndromes.

- Hereditary hemorrhagic telangiectasia should be considered in younger patients presenting with stroke or transient ischemic attack if the cause is unclear.

- In these patients, a thorough personal and family history of epistaxis and of respiratory and neurologic symptoms should be elicited.

- These patients should be closely examined for the presence of telangiectasias.

regular pulse rate, his blood pressure was $116 / 65 \mathrm{~mm} \mathrm{Hg}$, and his respiratory rate was 15 breaths per minute. He had finger and toe clubbing and several telangiectasias on his lips, tongue, gums and conjunctiva (Figure 1). The results of the rest of the clinical examination were normal.

Initial investigation showed that the patient had a normal hemoglobin level (151 [normal 120-160] g/L) and reduced oxygen saturation ( $90 \%$ [normal $\geq 95 \%$ ] on room air). The levels of his electrolytes, glucose, lipids and inflammatory markers were normal, as were the results of a hypercoagulable screen. Because we suspected pulmonary arteriovenous malformations, we measured the patient's arterial blood gases in the supine and standing positions. The patient had a supine $\mathrm{pH}$ of 7.4, a partial pressure oxygen of 60.2 (normal 80-100) $\mathrm{mm} \mathrm{Hg}$, a partial pressure carbon dioxide of 34.2 (normal $35-45) \mathrm{mm} \mathrm{Hg}$ and an oxygen saturation of $91 \%$. When standing, his $\mathrm{pH}$ level was 7.4, his partial pressure oxygen was 56.8 $\mathrm{mm} \mathrm{Hg}$ and his carbon dioxide level was $32 \mathrm{~mm} \mathrm{Hg}$ with oxygen saturation of $89 \%$. A shunt fraction of $21 \%$ was calculated by use of the $100 \%$ inspired oxygen breathing method (upper end of physiological shunt 5\%-8\%). Pulmonary arteriovenous malformations can lead to unexplained hypoxemia with further desaturation occurring when standing (orthodeoxia), as occurred in our patient. This finding is because of greater gravityinduced blood flow through basally situated pulmonary arteriovenous malformations which thereby increases the right-toleft shunt and hypoxemia. ${ }^{1}$ The results of cardiography and chest radiography were normal.

From the Department of Medicine, University of Alberta, Edmonton, Alta. 


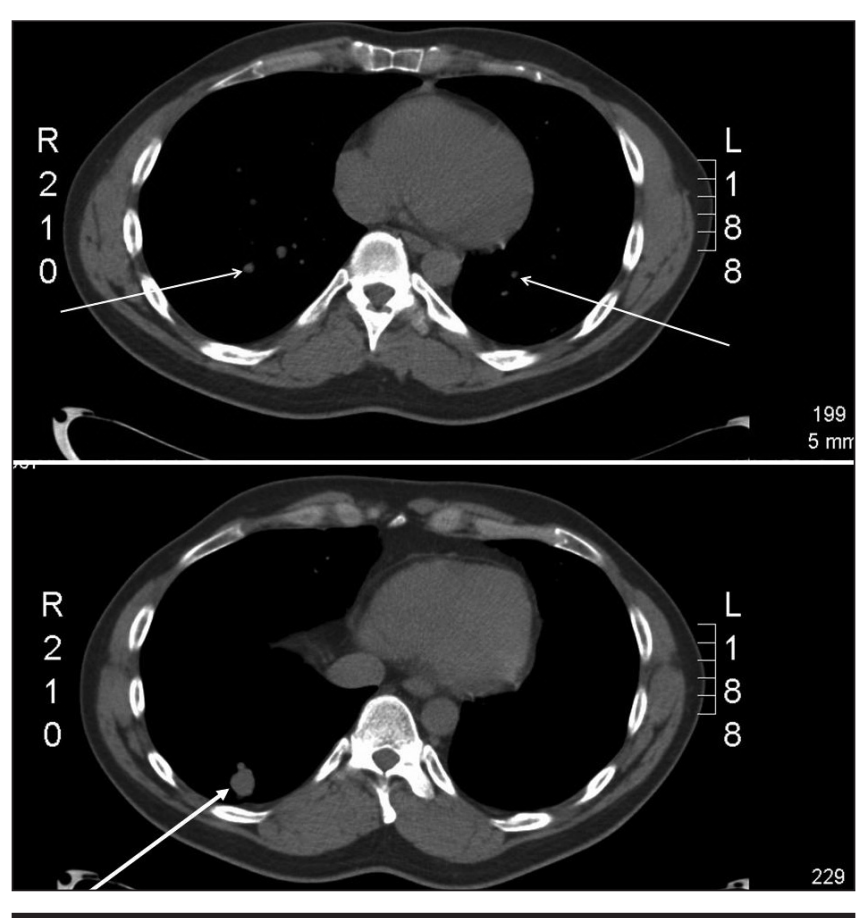

Figure 2: Computed tomogram of the patient's chest showing nodular soft-tissue densities (arrows), with the largest (lower panel) in the anterior basal region of the lower lobe with a feeding vessel of 8-10 $\mathrm{mm}$ in diameter.

Magnetic resonance imaging of the patient's brain showed an old cerebellar infarct on the right side, but there were no new infarcts, hemorrhages or malformations. The results of Doppler ultrasonography of his carotid arteries were normal. A transesophageal echocardiogram obtained with agitated saline showed microbubbles entering the left atrium from a direction suggestive of an extracardiac shunt.

A computed tomography chest scan without contrast showed multiple nodular soft-tissue densities scattered throughout both lungs, predominantly in the periphery. Many of the densities had a lobulated appearance (Figure 2). The largest was in the anterior basal region of the right lower lobe with a feeding vessel of $8-10 \mathrm{~mm}$ in diameter (Figure 2). This lesion, along with the 2 other large lesions in the right middle lobe and left upper lobe, represented the typical appearance of pulmonary arteriovenous malformations, each with a characteristic feeding artery and draining vein. At this point, a definitive diagnosis of hereditary hemorrhagic telangiectasia was made based on the presence of 3 of the 4 Curaçao criteria': history of epistaxis, presence of telangiectasias and presence of pulmonary arteriovenous malformations.

The largest pulmonary arteriovenous malformation in the right lower lobe was subsequently treated with coil embolization. The patient made a complete recovery and was discharged with a diagnosis of transient ischemic attack. He was followed-up in a hereditary hemorrhagic telangiectasia clinic. He had no recurrence of neurological symptoms over the following year and subsequently has had further therapeutic pulmonary embolizations with no complications. Genetic testing was not performed because the request for provincial funding was not approved.
Box 1: Neurological sequelae in hereditary hemorrhagic telangiectasia

\section{Stroke}

- Ischemic stroke or transient ischemic attack secondary to paradoxical embolus in the setting of pulmonary arteriovenous malformation. A vascular "steal" phenomenon is a much less likely cause.

- Hemorrhagic stroke secondary to cerebral arteriovenous malformation occurs in up to $10 \%$ of patients with hereditary hemorrhagic telangiectasia.

- Subarachnoid hemorrhage secondary to cerebral arteriovenous malformation or saccular aneurysm.

\section{Seizure}

- Probably caused by underlying cerebral arteriovenous malformation or a secondary complication of stroke or abscess.

\section{Cerebral abscess}

- Can be caused by paradoxical embolism of particulate septic material via a pulmonary arteriovenous malformation.

\section{Headache (migraine)}

- Caused by underlying cerebral arteriovenous malformation.

\section{Motor, sensory, bladder or bowel symptoms, muscle wasting}

- Caused by underlying spinal arteriovenous malformations.

\section{Porto-systemic encephalopathy}

- Caused by liver involvement and shunting through hepatic arteriovenous malformations.

\section{Discussion}

Our patient's diagnosis of hereditary hemorrhagic telangiectasia was made within a few days after presentation. However, he had previously been examined in hospital for neurological and gastrointestinal complaints, and hereditary hemorrhagic telangiectasia had not been recognized. Box 1 presents the neurologic sequelae of hereditary hemorrhagic telangiectasia. If the syndrome had been suspected earlier, appropriate intervention to treat his pulmonary arteriovenous malformations might have prevented his transient ischemic attack and, at the very least, allowed earlier testing and family screening.

We encourage physicians who encounter younger people with stroke or transient ischemic attack to ask questions about epistaxis and their family history of respiratory and neurological symptoms, and to closely examine the patient for telangiectasias as part of usual practice, especially when the cause is unclear.

This article has been peer reviewed.

Competing interests: None declared.

\section{REFERENCE}

1. Shovlin CL, Letarte M. Hereditary haemorrhagic telangiectasia and pulmonary arteriovenous malformations: issues in clinical management and review of pathogenic mechanisms. Thorax 1999;54:714-29. 